\title{
Using a sand wave model for optimal monitoring of a navigation depth
}

\author{
Michiel A.F. Knaapen \& Suzanne J.M.H. Hulscher \\ Water Engineering \& Management, University of Twente, Enschede, The Netherlands. \\ Meinard C.H. Tiessen \\ School of Civil Engineering, University of Nottingham, United Kingdom \\ Joris van den Berg \\ Numerical Analysis \& Computational Mechanics, University of Twente, Enschede, The Netherlands.
}

\begin{abstract}
In the Euro Channel to Rotterdam Harbor, sand waves reduce the navigable depth to an unacceptable level. To avoid the risk of grounding, the navigation depth is monitored and sand waves that reduce the navigation depth unacceptably are dredged. After the dredging, the sand waves slowly regain their original height. To reduce the high costs of surveying and dredging, the North Sea Service of the Department of Transport, Public Works and Water Management, is implementing a Decision Support System to reduce the required amount of surveys and provide optimal information on the necessity to dredge. Currently, the system predicts the growth of sand waves using a linear trend. The trend is determined from observations using a Kalman-filter including geo-statistical components to incorporate spatial dependencies. This works well for sand waves that are close to their maximum height. After dredging however, the sand wave height is far from its equilibrium and the growth rate is much higher, making the linear prediction worthless. Here we show that replacing the linear trend with a landau equation improves the predictions of the regeneration. Comparison shows that the landau equation predicts the crest evolution better than the linear equation for both undisturbed sand waves and dredged sand waves, with an root mean square error that is $25 \%$ less.
\end{abstract}

\section{INTRODUCTION}

With the increased navigation depth, required for modern ships, more and more harbor authorities are forced to dredge the entrance channels to their harbors. In the North Sea sand waves limit the depth of the navigation channel to Rotterdam Harbor, which makes dredging necessary. The guaranteed depth in this Eurochannel is 24 meter to allow for ships with a draught of $22.5 \mathrm{~m}$ or 75 feet. However, using the high tide, ships with a draught up to 26 meter can enter the harbor (Van De Gouwe 2003). Therefore the guaranteed navigation depth in the approach to the Eurochannel is 28.5 meter. The costs of repeated dredging are high and the authorities want to minimize these costs. To minimize the dredging costs, it is crucial to know the rate at which the sand waves regain their original shape.

For this purpose, the North Sea Service of the Department of Transport, Public Works and Water Management, is implementing a Decision Support System (DSS) to reduce the required amount of surveys and provide optimal information on the necessity to dredge. Currently, the system predicts the growth of sand waves using a linear trend (Wüst 2004). The trend is determined from observations using a Kalman-filter including geo-statistical components to incorporate spatial dependencies. This works well for sand waves that are close to their maximum height. Given the natural variations, the linearization is sufficiently accurate. After dredging however, the sand wave height is far from its equilibrium and the growth rate is much higher, making the linear prediction worthless.

Here, we test if the DSS to manage the navigation channel sea bed can be improved following the approach proposed by Knaapen (2005). In the Bisanseto Sea a field of sand waves has been topped off (Katoh et al. 1998) and there, the sand waves regenerated in several years time. Knaapen and Hulscher (2002) proved that this regeneration can be modelled using a Landau equation. In the North Sea navigation channel to Rotterdam Harbor, individual sand waves have been lowered. Here, we will show that the Landau model is valid to estimate the regeneration of the sand waves for such dredging strategy as well.

Offshore sand waves are elongated rhythmic bed features spaced about 200 to 500 meters apart, and several meters high. Sand waves are found in shallow tidal seas all over the world (Ludwick 1972; Boggs 


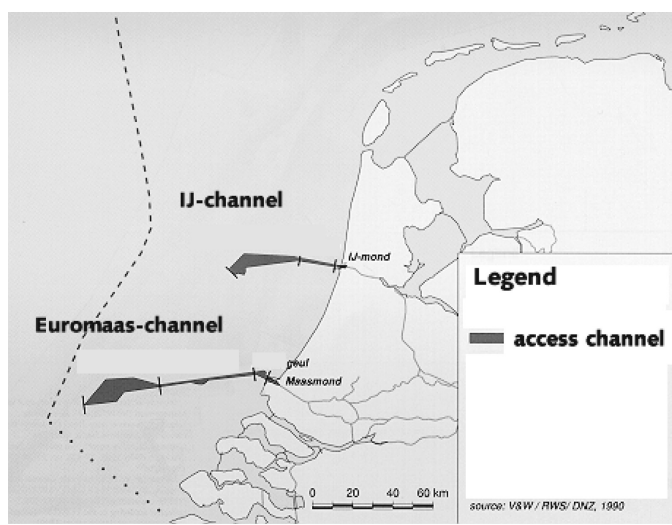

Figure 1. The approach to Rotterdam Harbor. The study area is found in the western part of the Euromaas channel. The length indicator in the picture covers $60 \mathrm{~km}$.

1974; Field et al. 1981; Aliotta and Perillo 1987; Harris 1988; Huntley et al. 1982; Ikehara and Kinoshita 1994; Katoh et al. 1998). Sand waves only occur in seas, where the tidal motion has to be strong, the maximum tidal velocity should be between 0.4-1 m/s (Stride 1982; Amos and King 1984). Furthermore, the bed should consist of non-cohesive sand (percentage silt less than 15\%, gravel less than 5\% (Terwindt 1971; Langhorne 1973; Bokuniewicz et al. 1977; Hulscher and Van Den Brink 2001).

It is assumed, that the sand waves are free instabilities of the system of an erodible bed and the tidal flow over it (Hulscher 1996; Komarova and Hulscher 2000; Gerkema 2000). It is shown that a stability analysis helps to explain the generation of sand waves. Furthermore, this approach can be used to estimate in which regions sand waves can be found (Hulscher and Van Den Brink 2001). The consequence of the sand waves being free instabilities is that they tend to recover after dredging. Németh (2003) shows that the stability models can simulate the growth to finite amplitude.

This paper has the following outline. First, the study area is described in section 2. In section 3 the approach to predict the regeneration of sand waves is explained. The testing data are described in section 4 . In section 5 the result of this approach are shown. Finally these results are discussed in section 6 .

\section{STUDY AREA}

The study area is located in the North Sea at latitude $51 \mathrm{~N}$ and longitude $3 \mathrm{E}(5750 \mathrm{~km}$ northing and 480 $\mathrm{km}$ easting in UTM ED50) at the entrance to the Euro Channel. The Euro Channel is the sea channel connecting the harbor of Rotterdam with the deeper parts

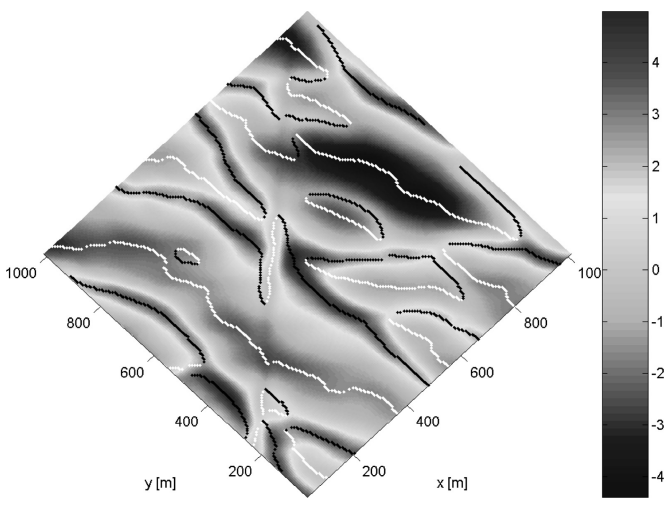

Figure 2. The results of the data reduction. The smooth gridded data are shown as a map, while the resulting wave crests and troughs are plotted as respectively black and white dots. The trench from North (top of figure) to South is the result of the recent dredging of a pipeline.

of the North Sea. The channel is about $60 \mathrm{~km}$ long and is orientated from east to west (see figure 2). In the offshore part crest of the sand waves have to be dredged to maintain the guaranteed navigation depth. In this area, two bed patterns are dominant: sand waves and megaripples. The sand waves are approximately stationary. The mean depth relative to mean sea level is 32 meter, but sand waves reduce the navigation depth to about 28 meter, which is the local guaranteed depth. The tidal range is 0.8 meter and the average tide has a maximum depth-averaged tidal velocity of about $0.8 \mathrm{~m} / \mathrm{s}$, but current velocities of over one meter can occur. The principal tidal axis has an approximately ca. 15 degrees clockwise angle with the north. The waves are moderate with a mean wave height of 1.3 meter, and only $10 \%$ of the waves exceed 2.5 meter (De Ronde et al. 1995).

\section{APPROACH}

To predict the growth of individual sand waves a number of techniques are combined. The basis of the approach is the work of Wüst (2004), who developed a Bayesian modelling approach for the sand waves. In the Bayesian approach, the height of individual sand waves is assumed to grow with constant speed. The growth speed is determined from the available measurements using a Kalman Filter. A geo-statistical relationship helps to reduce the impact of noise. This approach works fine for undisturbed sand waves. In the case of dredging, however, the sand wave dynamics change significantly. Therefore, a new model is proposed based on the Landau model of Knaapen and Hulscher (2002). As this model only describes the amplitude of the sand waves, the bathymetric data 


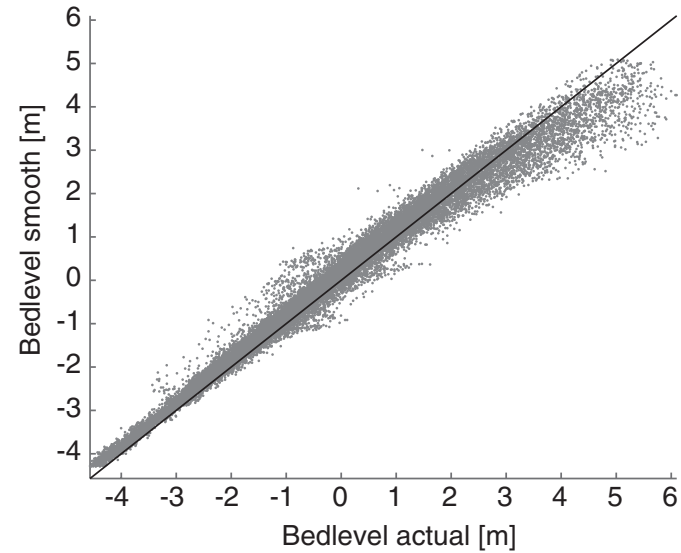

Figure 3. The relation between the raw data bed levels and the filtered bed levels. The filtering algorithm underestimates the crest. The magnitude is related to the occurrence of mega-ripples.

needs to be reduced to the crest positions. This has an additional advantage that it reduces the computational time considerably. For this purpose, the approach of Knaapen (2005) is followed.

\subsection{Data reduction}

The approach of Knaapen (2005) is based on the classical way to analyze rhythmic bed patterns. The locations of the crest and trough are compared to determine the length, height and other characteristics ( $\mathrm{O}^{\prime}$ Conner 1992). This approach has been proven to be robust and reliable. An automated procedure determines the crest and trough positions in both one-dimensional and twodimensional bathymetric data. For this purpose the data is filtered using a low-pass Bartlett window. This removes all noise related to mega-ripples and ripples. The crests can than be identified as the local maxima in the direction of the principle tidal axis, respectively. Due to the filtering procedure, this procedure results in a slight underestimation of the sand wave height (figure 3). This error is marginal however, compared to the noise of mega-ripples and can be corrected using a correction factor.

The shape characteristics of the sand waves can be deduced from the crest and trough positions of a single survey. The length of a sand wave is defined as the distance between the trough positions on opposite sites of the crest. The height is defined as the difference between the crest level and the mean of the two trough levels. Finally, the lee-stoss asymmetry is characterized as the difference of the distance between the crest and the lee trough and the distance between the crest and the stoss trough divided by the sand wave length.

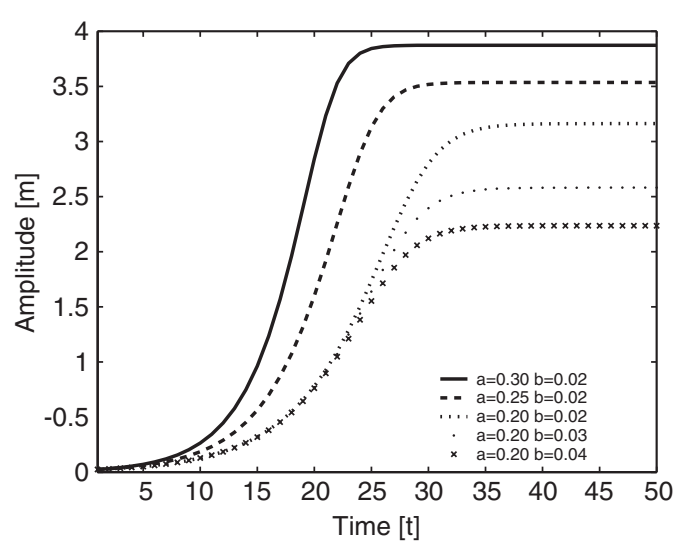

Figure 4. The sand wave growth according to the Landau model using several parameter combinations that are within the range of realistic values for North Sea conditions.

\subsection{Landau equation}

The Landau equation has been developed to describe the development of unstable waves in a variety of physical systems in which turbulence occurs. This equation describes the development of patterns from their initial stages until their equilibrium state (Doelman 1990). Knaapen and Hulscher (2002) shows that the growth of sand waves can also be described by a normalized form of the Landau equation:

$\frac{\partial A}{\partial t}=\alpha A+\beta A^{3}$.

This equation (1) describes the development of the amplitude of the sand wave $(A)$ in time $(t)$. The term $(\alpha A)$ describes the initial exponential growth for positive $\alpha$. The second term on the right hand side describes the non-linear growth. For small values of the amplitude, the non-linear term is small compared to the linear growth. With increasing amplitude, the non-linear term becomes more dominant, resulting in a dampening of the exponential growth towards an equilibrium value $A_{\infty}=\sqrt{-\frac{\alpha}{\beta}}$, provided that $\beta$ is negative.

To translate the amplitude to a water depth $z_{b}$ the sand wave height is added to an undisturbed depth $D$ :

$z_{b}=D-2 A$.

Figure 4 shows the Landau curve for some values of the linear growth rate $\alpha$ and the damping parameter $\beta$. The initial state vector $x 0$ were chosen to be $\alpha=0.125$ and $\beta=-0.0083$, whereas $D_{0}$ an $A_{0}$ are determined from the first measurement survey. 


\subsection{Extended Kalman filter}

A Kalman filter is a mathematical procedure to improve predicting qualities of a model. Based on the difference between the model prediction of a system and some measurements, it determines the most probable state of that system (Jazwinski 1970). The Kalman filter is based on a predictor-corrector algorithm. Traditionally, Kalman filters are linear (Kalman 1960). In order to be able to use the non-linear Landau equation, an extended Kalman filter is used (Jazwinski 1966).

Given the state estimate $x_{i}$, which in our case is defined as:

$x_{i}=[\alpha, \beta, A, D]^{T}$,

the first step is a model prediction following the nonlinear function $f\left(x_{i}\right)$ :

$$
\begin{aligned}
x_{i+1} & =f\left(x_{i}\right)+w_{i} \\
& =\left[1,1,\left(1+\alpha \Delta t+\beta \Delta t A_{i}^{2}\right) A_{i}, 1\right]^{T}+w_{i}
\end{aligned}
$$

which assumes that only the sand wave amplitude changes, following the Landau equation, with an added noise $w_{i}$, which has a normal distribution with zero mean and covariance $W_{i}$. It is then assumed that the model error changes linearly due to this prediction:

$P_{i+1}=F_{i} P_{i} F_{i}+W_{i}$

in which $P_{i}$ is the estimated covariance of the state estimate $x_{i} . F_{i}$ is the update of that covariance, which is equal to $f\left(x_{i}\right)$ if the latter is a linear function. In our case part of the prediction is non-linear: the change in the amplitude. In this case the changes in the error statistics can only be estimated. Therefore, in the extended Kalman filter, the real changes in the covariance are approximated using the first order linearization of $f\left(x_{i}\right)$ to the elements of the state estimate $x_{i}$ :

$F=\left[\begin{array}{cccc}1 & 0 & 0 & 0 \\ 0 & 1 & 0 & 0 \\ \Delta t A_{i} & \Delta t A_{i}^{3} & 1+\Delta t\left(\alpha+3 \beta A_{i}^{2}\right) & 0 \\ 0 & 0 & 0 & 1\end{array}\right]$

At some point in time $t_{n}$ a measurement is available:

$$
\begin{aligned}
d_{n} & =M x_{i}+r_{n} \\
& =\left[\begin{array}{llll}
0 & 0 & -2 & 1
\end{array}\right] x_{i}+r_{n}
\end{aligned}
$$

in which $M$ is the function that describes the measuring system leading to the measurement $d_{n}$ and $r_{n}$ is the measurement noise with zero mean and covariance $R_{n}$. At that moment the model prediction is compared to the measurements. In general, there will be a difference between the two. This difference is used to correct the state estimate based on the information on the model error and the measurement error:

$x_{i}^{c}=x_{i}+K_{i}\left(z_{i}-M x_{i}\right)$.

The Kalman gain $K_{i}$ compares the accuracy of the model prediction with the accuracy of the measurements and is determined based on the covariances of the measurement and the predicted value:

$K_{i}=P_{i}^{T} M^{T}\left(M P_{i} M^{T}+R_{n}\right)^{-1}$

The final calculation in the corrector phase is the update of the covariance of the state estimate, which will be reduced due to the additional information:

$P_{i}^{c}=\left(I-M K_{i}\right) P_{i}$

\section{TUNING AND TESTING OF THE KALMAN FILTER}

Before, the Kalman filter can be used to predict the bed changes in the Euro channel, it's covariance matrices have to be determined. Furthermore, we have to verify that the Kalman filter can be used to tune a Landau equation.

\subsection{Covariance parameters in the Kalman filter}

The Kalman filter uses covariance matrices to determine the accuracy of the model predictions and the measurements. The covariances of the measurements $R$ and of the predictions $Q$ need to be prescribed, as well as the covariance $P_{0}$ describing the accuracy of the initial estimate of the state vector. Here we describe the settings that are used for these covariances.

Within the prediction system, all errors sources are assumed to be independent. In that situation the prescribed covariance matrices reduce to diagonal matrices with the variances on the diagonal. This does not imply that the prediction errors are independent. Due to the covariance prediction (equation 5) and the covariance measurement update (equation 10) the errors interact, which is represented by the elements off the diagonal in the prediction covariance matrix.

The measurement noise $R$ is determined from the bathymetric surveys. The difference between the filtered data and the raw data shows that the noise due to ripples and mega-ripples has a standard deviation of is 0.55 meter. Furthermore, the measurement system (echosounder and GPS) has an error with a 0.25 meter standard deviation (Knaapen et al. 2005). This value is confirmed by the variation of the mean water depth. The total variance is set at the sum of the variances of these errors: $0.37 \mathrm{~m}^{2}$. 
The exact values of the variance of the initial state estimate are relatively unimportant. As long as one chooses them large enough, the Kalman filter will adjust the state covariances to acceptable levels. If the initial variances are chosen too small e.g. much smaller than the variances of the measurement, the Kalman filter will ignore the measurements and rely on the model predictions. The variances of the initial parameters estimates were set at:

$P_{0}=\left[\begin{array}{cccc}0.125 & 0 & 0 & 0 \\ 0 & 6 \cdot 10^{-6} & 0 & 0 \\ 0 & 0 & 0.3 & 0 \\ 0 & 0 & 0 & 0.08\end{array}\right]$

in which the variances of the amplitude and the undisturbed water depth are determined from the errors in the measurement system. The variances of the model parameters agree with a standard deviation that is larger than the initial parameter value $(\alpha)$ and about $25 \%$ of the initial parameter value $(\beta)$.

Finally, the model error is defined as being a fraction of the covariance matrix of the state estimates following (West):

$W_{i}=F_{i} P_{i} F_{i}^{T} \frac{1-W_{d f}}{W_{d f}}$

in which $W_{d f}$ is a diagonal matrix with on the axis 0.98 , except for the forth diagonal element, which is set at 0.99 . This implies that the prediction of the mean depth is more accurate than the predictions of the amplitude and the parameters.

\subsection{Testing the Kalman filter}

The Kalman filter is tested using an artificial data set. With a given parameter set, the Landau model is ran and its output stored. Next, this output is given to the Kalman filter as 'measurements'. If the Kalman filter is working properly, it should be able to recover the parameter settings from these 'measurements'. Figure 5 shows that the Kalman filter works perfectly. The parameter estimates smoothly converge to the parameter values used in the original Landau model that generated the 'measurements'. Figure 6 shows that, although the Kalman filter is sensitive to the noise, but still manages to retrieve the original parameter settings. For this test, a random noise has been added to the measurements produced by the landau model. This noise has zero mean and standard deviation of 0.5 meter. This is comparable with the noise resulting from mega-ripples. Initially, the Kalman filter has difficulty with the noise. The confidence in the predictions is less than the reliability of the measurements. However, as time progresses, the estimates become more accurate, giving more weight to the model runs,
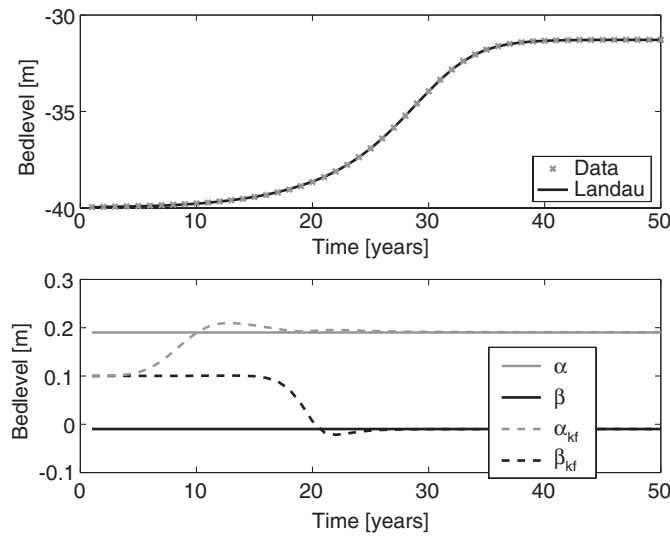

Figure 5. Testing the Kalman filter. top: the predicted water depth compared with the original model results. Bottom: the parameter estimates ( $\alpha_{k f}$ and $\beta_{k f}$ ) converge to their original value ( $\alpha$ and $\beta$ ).
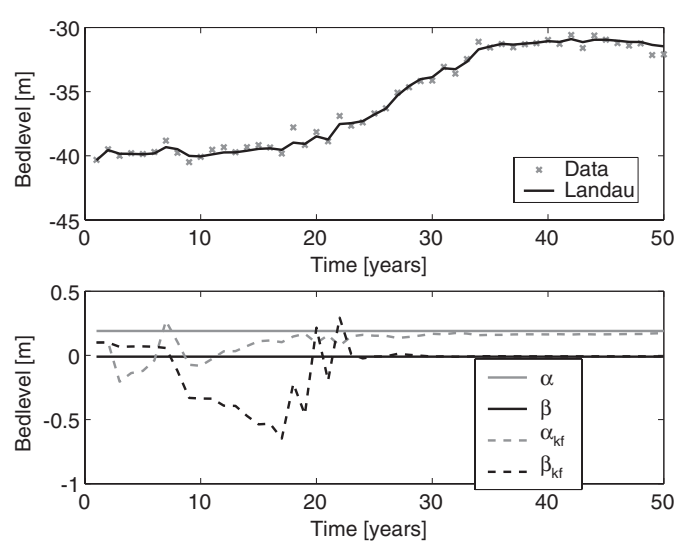

Figure 6. Testing the Kalman filter with noise (standard deviation $0.5 \mathrm{~m}$ ). Top: the predicted water depth compared with the original model results. Bottom: after some initial problems, the parameter estimates $\left(\alpha_{k f}\right.$ and $\left.\beta_{k f}\right)$ converge to their original setting ( $\alpha$ and $\beta$ ).

and the Kalman filter does find the correct parameter values. These results show, that it is possible to tune a Landau model using a Kalman filter algorithm.

\subsection{Test data}

The proposed approach is tested against several sand waves, both undisturbed and dredged. From these sand waves, two are selected to visualize the quality of the combination of the Kalman filter and the Landau equation. The first one is a large sand wave that is just below the critical depth, but has not been dredged. This will test the predictions on the natural dynamics 


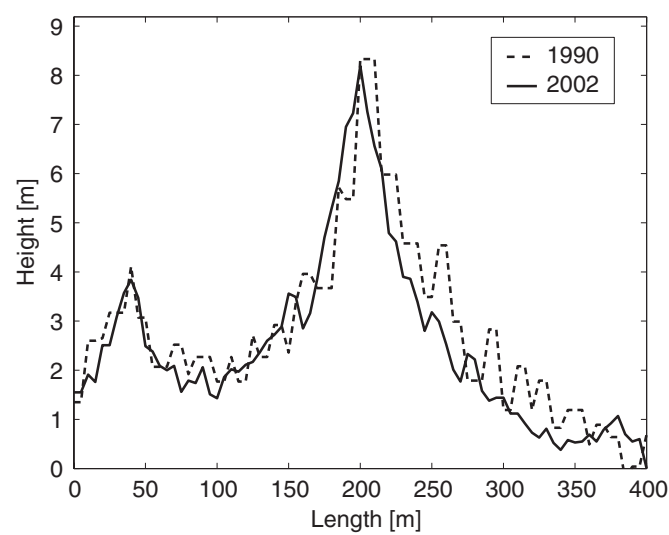

(a) Undisturbed sand wave

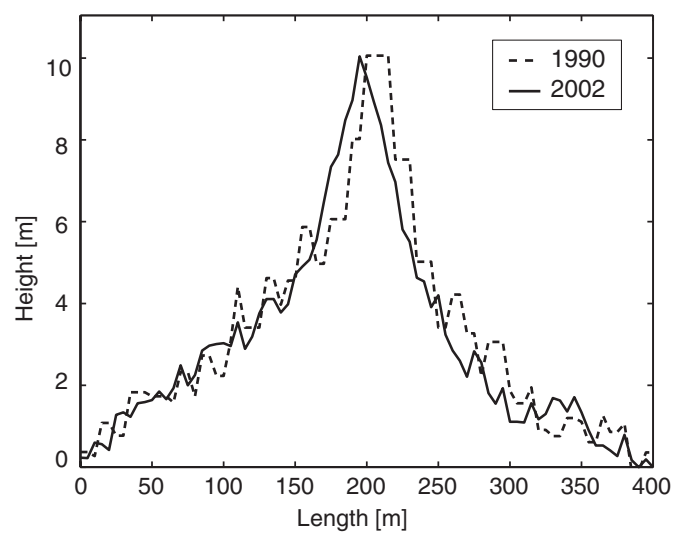

(b) dredged sand wave

Figure 7. profile of 7(a) an undisturbed and 7(b) a dredged sand wave used for testing.

of the sand waves. Figure 7(a) shows the cross-profile in two years. The second data set crosses the critical depth and has been dredged twice in 1992 and 1995. Figure 7(b) shows the cross-section of this sand wave.

\section{RESULTS}

\subsection{Undisturbed sand wave}

As a first test against field data, both the Landau model and the linear model are applied to undisturbed sand waves. Both models are applied to the data sets of sand waves that have not been dredged yet. For comparison, the error statistics of both models are calculated. The results in the prediction of the development of an undisturbed sand wave development is shown in Figure 8 . The accuracy of the earlier data is still limited. But even without this error the initial settings are not in agreement with the data. The model predicts a growing

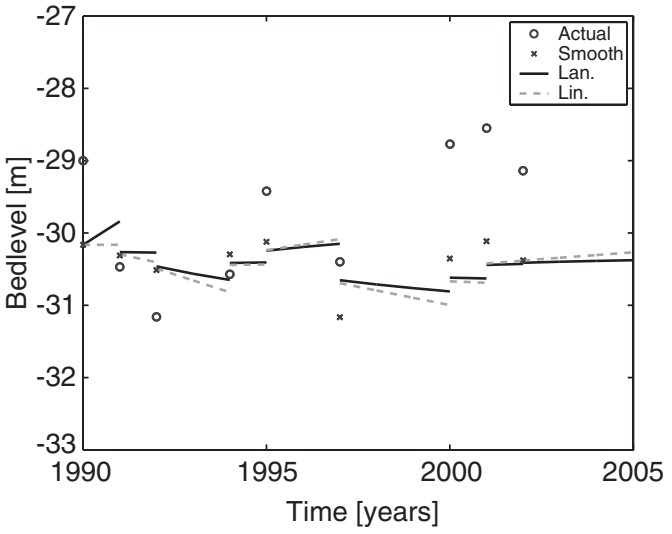

Figure 8. The development of the undisturbed sand wave in time and the estimates with both the Landau model and the linear model.

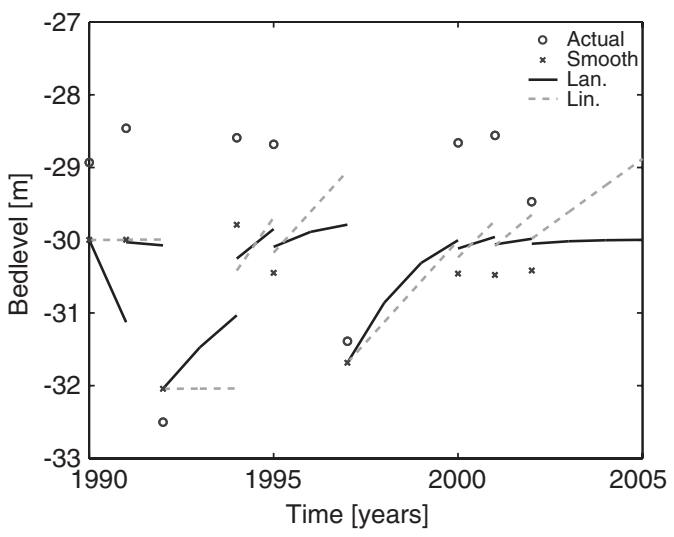

Figure 9. The development of the dredged sand wave in time and the estimates with both the Landau model and the linear model.

sand wave in the first year, whereas the sand wave is fairly stable.

As the changes in the sand wave are small and mostly within the range of the measurement uncertainty, both models have difficulty adjusting to the situation. The Kalman filter has plenty of freedom to adjust the sand wave height and the undisturbed water depth $D$ with little parameter change. The landau model, however, does estimate the bed levels better than the linear model.

\subsection{Dredged sand wave}

The main goal of this project is to improve the Decision Support System of the navigation channel sea bed in the predicting of sand wave development after dredging. Figure 9 shows the model results of a sand wave, 
that has been dredged twice since 1990. In 1992 and in 1995 the crest of the sand wave is lowered. This human interference cannot be described by both models, due to a lack of information about the dredging operations. Therefore, the models restart their predictions from the first observed water level after the dredging operation.

After the dredging operation of 1992, the difference between the functioning of the Landau equation and the linear equation is striking. The Landau model correctly predicts a high increase in sand wave amplitude, directly after dredging, while the linear model predicts a steady development. This difference shows that the Landau model does describe the recovery after dredging operations better than the linear model.

One year after the dredging operation the sand wave is totally recovered and at the same height as before the dredging operation. Now the linear model predict a strong further growth, whereas the Landau model does show a relaxation to a stable bed level. This level is a bit conservative, but well within the range of the measurement uncertainty related to the mega-ripples.

The linear model is completely messed up by the dredging operations. The linear trend is being tuned for the situation after dredging operations. After the first dredging, it found an acceptable growth rate within two years. The first test of this rates takes place immediately after the second dredging operation. As this prediction is correct, the trust in the trend is very high. Consequently, the Kalman filter is unable to adapt to the stable sand wave height in the remaining years.

Again, the initial parameter settings of Landau model are incorrect. This results in an incorrect predicted initial decrease in sand wave height predicted. In this situation, the initial sand wave height is bigger than the maximum height assumed by the Landau model. After adjustment to this error, the Landau model reproduces the bed level dynamics well.

\subsection{Overview}

To compare and quantify the Landau model capabilities with the linear model capabilities, the models have been applied to more locations. In total 30 locations of undisturbed sand waves, and 24 locations of dredged sand waves are tested. However, due to limited information on dredging operations in the past, these 24 locations are located at only eight different sand waves. This does result in some correlation between these 24 locations with dredged sand waves.

Table 1 shows the root mean square differences between the predicted model bed levels, without the measurement update and the measured values. To remove the tuning problems from the error estimates, the first 6 measurements are excluded from the error estimates.

For the situation of undisturbed sand waves, the Landau equation can predict the sand wave
Table 1. Root mean square prediction error for the Landau equation and the linear equation for both undisturbed and dredged sand waves, after 2000.

\begin{tabular}{lll}
\hline Model & Undisturbed & Dredged \\
\hline Landau & $0.26 \mathrm{~m}$ & $0.37 \mathrm{~m}$ \\
Linear & $0.32 \mathrm{~m}$ & $0.45 \mathrm{~m}$ \\
\hline
\end{tabular}

Table 2. The initial and final parameter setting as well as the root mean square error (RMSE) of the initial guess.

\begin{tabular}{llc}
\hline & $\alpha$ & $\beta$ \\
\hline Initial & 0.125 & -0.0083 \\
\hline Undisturbed & & \\
Final & 0.054 & -0.009 \\
RMSE & 0.38 & 0.0128 \\
Dredged & & -0.009 \\
Final & 0.139 & 0.0286 \\
RMSE & 0.64 & \\
\hline
\end{tabular}

development about $23 \%$ better than the linear equation. For the situation of dredged sand waves, the improvement is about $22 \%$.

The Kalman filter has adapted the initial parameter values to improve predicting capabilities for both equations. The initial estimation of the parameter values was expected to be close to the occurring values. In both examples displayed above, the figures suggested that the estimated parameter values were rather inaccurate.

To analyze the quality of the initial parameter estimations, the initial and final parameter values are compared. Table 2 shows the initial values and the final values as well as the root mean square difference between them. From these values it is clear that the differences in the initial value of the linear growth rate ( $\alpha$ are large, whereas the value of the non-linear dampening term is small. The initial settings overestimate the asymptotic sand wave height.

\section{CONCLUSIONS AND DISCUSSION}

The Landau equation is a clear improvement to the decision support system for dredging and monitoring management of the Approach to Rotterdam Harbor. Both the natural behavior and the regeneration of sand waves after dredging are predicted more accurately. Moreover, the dynamics of the Landau equation are in line with the theoretical dynamics.

The non-linear model requires the use of the extended Kalman filter. The linear approximation of 
the error statistics in the extended Kalman filter results in a near optimal state, as long as the time intervals are small enough for this linear approximation. In our case, the time step between measurement surveys never more than a couple of years. This period is small enough for the extended Kalman filter to give reliable results.

The data reduction procedure of Knaapen (2005) does reduce to amount of information enormously, without losing essential information on the navigation depth. A negative side effect of the data filtering procedure used in this project is that the average sand wave height at the crest is lowered with 0.61 meter. This could be repaired either, by adding a correction term on top of the crests, or by replacing the filtered crest level by the absolute maximum bed level around this crest position. This would result in a conservative estimate of the navigation depth.

Without the geo-statistical information analysis used by (Wüst 2004), the noise in the results is unacceptably high. Therefore, this analysis should be combined with the Landau model. At first sight, the data reduction procedure compromises the geo-statistics procedure, as it removes a lot of spatial information. However, the most important information is still present, being the level of the neighboring crest points. However, information on the mega-ripples might be reduced, which could be essential information to reduce the noise.

The sand wave regenerate much faster than expected. It never to more than a few years for the sand waves to recover. Occasionally, the sand waves returned to their natural state within a year. This is much faster than the theoretical growth rates (Hulscher 1996; Németh 2003). It is also considerably faster than the regeneration observed by Katoh et al. (1998) in the Bisanseto sea. One explanation may be that the removal of a single crest does not effect the flow pattern significantly. The bathymetry is out of balance with the flow field. As a result, increased sediment deposition in the dredging area is expected, from theory based on a growth of a group of sand waves.

The parameters that are based on local conditions and existing theory are inaccurate. The Kalman filter has to correct the initial parameters significantly.

The administration of dredging operations has never intended to facilitate research. As a result it is difficult to find information on the performed dredging. It is especially difficult to determine exact location, the dredging method that is used and the dredging amounts from the archives. To get better insight in the regeneration of the dredged sand waves, it would be favorable to archive future dredging operations on exact location, dredging quantities, and the location on which the material is dumped. A large step forward is the post-dredging survey that is now the practice in the approach channel to Rotterdam Harbor.

\section{ACKNOWLEDGEMENTS}

The research described in this paper, is funded by the Technology Foundation STW, applied science division of NWO and the technology program of the Ministry of Economic Affairs (project number TWO.5805: Modelling of Spatial and Temporal Variations in Offshore Sandwaves: Process-Oriented vs. Stochastic Approach). We acknowledge the feedback of Dr. A.A. Németh and Ir. H. Wüst. Furthermore, we thank the the North Sea Directorate of the Netherlands Department of Waterways and Public Works for their cooperation and for making the measurements available.

\section{REFERENCES}

Aliotta, S. and G. M. E. Perillo (1987). A sand wave field in the entrance to Bahia Blanca estuary. Marine geology 76, $1-14$.

Amos, C. L. and E. L. King (1984). Beforms of the Canadian Eastern Seabord: A comparison with global occurrences. Marine Geology 57, 167-208.

Boggs, S. (1974). Sand wave fields in Taiwan Straight. Geology 2, 251-253.

Bokuniewicz, H. J., R. B. Gordon, and K. A. Kastens (1977). Form and migration of sand waves in a large estuary, long island sound. Marine Geology 24, 185-199.

De Ronde, J. G., J. G. A. Van Marle, A. P. Roskam, and J. H. Andorka Gal (1995). Golfrandvoorwaarden langs de nederlandse kust op relatief diep water. Technical Report RIKZ-95.024, Rijkswaterstaat, Rijksinstituut voor Kust en Zee/RIKZ, Den Haag.

Doelman, A. (1990). Finite-dimensional models of the Ginzburg-Landau equation. Nonlinearity, 231-250.

Field, M. E., C. H. Nelson, D. A. Caccione, and D. E. Drake (1981). Sand waves on an epicontinental shelf: Northern Bering Sea. Marine Geology 42, 233-258.

Gerkema, T. (2000). A linear stability analysis of tidally generated sand waves. Journ. of Geoph. Res. 417, 303-322.

Harris, P. T. (1988). Sediment, bedforms and bedload transport pathways on the continental shelf adjacent to Torrres Strait, Australia-Papua New Guinea. Continental Shelf Research 8(8), 979-1003.

Hulscher, S. J. M. H. (1996). Tidal-induced largescale regular bed form patterns in a three-dimensional shallow water model. Journal of Geophysical Research 101(C9), 20, 727-20, 744.

Hulscher, S. J. M. H. and G. M. Van Den Brink (2001). Comparison between predicted and observed sand waves and sand banks in the north sea. Journal of Geophysical Research 106(C5), 9327-9338.

Huntley, D. A., J. M. Huthnance, M. B. Collins, C.-L. Liu, R. J. Nicholls, and C. Hewitson (1982). Hydrodynamics and sediment dynamics of North Sea sand waves an sand banks. Phil. Trans. R. Soc. London 4, 79-99.

Ikehara, K. and Y. Kinoshita (1994). Distribution of subaqueous dunes on the shelf of Japan. Marine Geology 120, 75-87.

Jazwinski, A. H. (1966). Filtering for nonlinear dynamical systems. IEEE transactions on automatic control AC11(4), 765-end. 
Jazwinski, A. H. (1970). Stochastic Processing and Filter Theory. New York: Academic Press.

Kalman, R. (1960). A new approach to linear filter and prediction theory. Journal of Basic Engineering 82D, 35-45.

Katoh, K., H. Kume, K. Kuroki, and J. Hasegawa (1998). The development of sand waves and the maintenance of navigation channels in the Bisanseto Sea. In Coastal Engineering '98, pp. 3490-3502. ACSE.

Knaapen, M. A. F. (2005). Sand wave migration predictor based on shape information. Journal of Geophysical Research - Earth Surface 110. In Press.

Knaapen, M. A. F. and S. J. M. H. Hulscher (2002, September). Regeneration of sand waves after dredging. Coastal Engineering 46(4), 277-289.

Knaapen, M. A. F., C. N. Van Berge Henegouw, and Y. Y. Hu (2005). Quantifying bedform migration using multi-beam sonar. Geo-Marine Letters. Accepted for publication.

Komarova, N. L. and S. J. M. H. Hulscher (2000). Linear instability mechanics for sand wave formation. J. of. Fluid Mechanics 413, 219-246.

Langhorne, D. N. (1973). A sand wave field in the outer Thames estuary, great Britain. Marine Geology 14(2), 129-143.

Ludwick, J. C. (1972). Migration of tidal sand waves in chesapeake bay entrance. In D. Swift, D. Duane, and O. Pilkey
(Eds.), Shelf Sediment Transport: Process and Pattern, Stroudsburg, Pa., pp. 377-410. Dowden, Hutchinson and Ross, Inc.

Németh, A. A. (2003). Modelling offshore sand waves. $\mathrm{Ph}$. D. thesis, The Netherlands.

O'Conner, B. A. (1992). Prediction of seabed sand waves. In W. Patridge (Ed.), Computer modelling of seas and coastal regions, pp. 322-338. Southampton: Computational Mechanics Publishers.

Stride, A. H. (Ed.) (1982). Offshore Tidal Sands: Processes and Deposits. London: Chapman and Hall.

Terwindt, J. H. J. (1971). Sand waves in the Southern Bight of the North Sea. Marine Geology 10, 51-67.

Van De Gouwe, J. (2003). Instandhoudingsplan bodem vaarroute naar Rotterdam. Technical report, North Sea Directorate of the Netherlands Department of Transport, Public Works and Water Management, Rijswijk.

West, M. Bayesian forecasting. In S. Kotz, C. B. Read, and D. Banks (Eds.), Encyclopedia of Statistical Sciences. New York: Wiley.

Wüst, H. (2004, April). Data-driven probabilistic predictions of bathymetry. In S. Hulscher, T. Garlan, and D. Idier (Eds.), Marine Dynamics II. University of Twente. 
